\title{
Propensity-Score Matched Analysis of the Efficacy of Maintenance/Continuous Therapy in Newly Diagnosed Patients With Multiple Myeloma: A Multicenter Retrospective Collaborative Study of the Japanese Society of Myeloma
}

Shuji Ozaki ( $\square$ ozaki@tph.gr.jp )

Tokushima Prefectural Central Hospital https://orcid.org/0000-0002-9808-0752

Hiroshi Handa

Gunma University

Hiromi Koiso

Gunma University

\section{Takayuki Saitoh}

Gunma University

Kazutaka Sunami

Okayama Medical Center

Tadao Ishida

Japanese Red Cross Medical Center

Kenshi Suzuki

Japanese Red Cross Medical Center

Tomoko Narita

Nagoya City University

Shinsuke lida

Nagoya City University

Yuichi Nakamura

Saitama Medical University

\section{Kazuhito Suzuki}

Jikei University Kashiwa Hospital

Noriko Nishimura

Cancer Institute Hospital of the Japanese Society for Cancer Research

Hirokazu Murakami

Gunma University of Health and Welfare: Gunma Iryo Fukushi Daigaku

Kazuyuki Shimizu

Higashi Nagoya National Hospital 


\section{Research Article}

Keywords: multiple myeloma, revised International Staging System, maintenance therapy, continuous therapy, autologous stem cell transplantation

Posted Date: April 13th, 2021

DOl: https://doi.org/10.21203/rs.3.rs-401187/v1

License: (c) (i) This work is licensed under a Creative Commons Attribution 4.0 International License.

Read Full License

Version of Record: A version of this preprint was published at Journal of Cancer Research and Clinical Oncology on June 2nd, 2021. See the published version at https://doi.org/10.1007/s00432-021-03668-6. 


\section{Abstract}

Maintenance/continuous therapy is considered a standard of care for both transplant-eligible and ineligible patients with multiple myeloma (MM). However, long-term benefits of such therapy have not yet been clarified in the context of clinical practice. We retrospectively analyzed the efficacy of maintenance/continuous therapy in newly diagnosed MM patients using the cohort data by propensityscore matching based on age, gender, revised International Staging System (R-ISS) stage, and implementation of transplantation to reduce the bias due to confounding variables. Among 720 patients, 161 were identified for each of the maintenance and no maintenance groups. Maintenance/continuous therapy employed immunomodulatory drugs $(n=83)$, proteasome inhibitors $(n=48)$, combination of both $(n=29)$, or dexamethasone alone $(n=1)$. Progression-free survival (PFS) was significantly prolonged in the maintenance group compared with the no maintenance group (median, 37.7 and 21.9 months, $p=$ 0.0002 , respectively). Prolongation of PFS was observed in both transplanted and non-transplanted patients $(p=0.017$ and $p=0.0008$, respectively), with standard risk $(p<0.00001)$, R-ISS stage I $(p=0.037)$ and stage II $(p=0.00094)$, and those without obtaining complete response $(p=0.0018)$. There was no significant difference in overall survival $(p=0.19)$, but it appeared to be better in non-transplanted patients by continuous therapy. These results support the usefulness of maintenance/continuous therapy in the management of MM.

\section{Introduction}

Multiple myeloma (MM) is a plasma cell neoplasm characterized by the presence of monoclonal immunoglobulin (lg) in serum and/or urine, clonal bone marrow plasmacytosis, and clinical symptoms related to hypercalcemia, renal insufficiency, anemia, and bone lesion (CRAB features), as well as other myeloma-defining events (International Myeloma Working Group, 2003; Rajkumar et al, 2014). MM is a heterogeneous disease, and the survival outcome varies considerably depending on the presence of patient-related, disease-related, and treatment-related risk factors (Rajkumar, 2020).

Recently, significant advances have been made in the treatment of MM. In particular, treatment incorporating novel agents and autologous stem cell transplantation (ASCT) have significantly improved survival of MM patients (Kumar et al, 2014; Kumar et al, 2008; Ozaki et al, 2015). Besides, as a treatment option, maintenance or continuous therapy after induction therapy has been evaluated in both transplanteligible and -ineligible MM patients in clinical trials and routine practice. This treatment strategy has been developed to improve and sustain disease response, ultimately bringing a functional cure by long-term continuous treatment, and has now been recognized as a new paradigm in the management of MM (Dimopoulos et al, 2020a).

Randomized controlled studies of maintenance therapy after ASCT have demonstrated the efficacy and feasibility of immunomodulatory drugs (IMiDs) such as lenalidomide and thalidomide as well as proteasome inhibitors (PIs) comprising bortezomib and ixazomib (Attal et al, 2012; Dimopoulos et al, 2019; Goldschmidt et al, 2018; Jackson et al, 2019; McCarthy et al, 2012; Morgan et al, 2012; Palumbo et 
al, 2014b; Sonneveld et al, 2012). Most studies have witnessed the benefits of maintenance therapy in progression-free survival (PFS) but not in overall survival (OS), while meta-analyses did show the benefits also in OS (McCarthy et al, 2017; Morgan et al, 2012). In transplant-ineligible patients, randomized studies have shown the benefits of continuous therapy using lenalidomide, thalidomide, and/or bortezomib, or ixazomib in PFS (Dimopoulos et al, 2020b; Palumbo et al, 2014a; Palumbo et al, 2010; Palumbo et al, 2012). Meta-analysis of these studies involving non-transplanted patients has demonstrated the significant prolongation of PFS, PFS2, and even OS (Palumbo et al, 2015b). Therefore, recent studies are turned out to be designed to evaluate the effects of continuous therapy with lenalidomide, bortezomib, ixazomib, and/or daratumumab subsequent to backbone therapy until disease progression (Benboubker et al, 2014; Durie et al, 2017; Durie et al, 2020; Facon et al, 2018; Facon et al, 2019; Mateos et al, 2020; Mateos et al, 2018).

The clinical benefits of lenalidomide or bortezomib as post-ASCT maintenance therapy have been investigated in routine clinical practice as well (Chakraborty et al, 2018; Huang et al, 2018; Jagannath et al, 2018; Sivaraj et al, 2017). However, these studies were observational and the influence of selection bias due to confounding factors for application of maintenance therapy in study patients cannot be ruled out. Moreover, the optimal drug combination as maintenance for suitable patient population, especially in transplant-ineligible patients, remains to be clarified in routine clinical practice.

Here, we conducted a retrospective cohort study of MM patients treated between 2013 and 2016 at 32 hospitals of the Japanese Society of Myeloma (JSM). We evaluated the efficacy of maintenance/continuous therapy for both transplant-eligible and -ineligible MM patients using the propensity-score matched analysis to reduce the bias due to confounding factors for estimating treatment effect between those who had received maintenance versus others who had not received such treatment.

\section{Patients And Methods}

\section{Patients}

The JSM retrospectively collected clinical data from 720 patients who were diagnosed and treated at 32 affiliated hospitals between January 2013 and December 2016 and had with at least stable disease response to initial treatment. Baseline demographics, clinical and laboratory data including fluorescence in situ hybridization (FISH) analysis, and details regarding induction and maintenance/continuous therapy and the initial response were assessed. This study was conducted in accordance with the institutional guidelines with approval of the Ethics Committee/Institutional Review Board of Gunma University.

\section{Diagnosis and stage}

The diagnosis of MM was made according to the International Myeloma Working Group (IMWG) criteria (2003) and the clinical stage of MM was determined based on the revised International Staging System 
(R-ISS) as previously published (Palumbo et al, 2015a). For risk stratification, $t(4 ; 14), t(14 ; 16)$ and/or del(17p) were considered as high risk. Patients with asymptomatic (smoldering) MM and primary amyloidosis were excluded.

\section{Treatment}

Treatment decision for induction therapy, consolidation with ASCT, and maintenance therapy after ASCT in transplant-eligible patients, or that for continuation of each treatment phase in transplant-ineligible patients was made at the discretion of a physician-in-charge according to the treatment policy of each facility. ASCT was conducted with high-dose melphalan conditioning followed by peripheral blood stem cell transplantation according to the institutional protocol and was regarded as initial therapy only when it was performed upfront after induction therapy. Treatment response was assessed according to the uniform response criteria reported by the IMWG (Durie et al, 2006).

\section{Propensity-score matched analysis}

We implemented 1:1 matching for the maintenance group and no maintenance group to evaluate solely the efficacy of maintenance/continuous therapy in the study cohort. Patients were stratified according to clinical and laboratory parameters such as age, gender, R-ISS stage, and implementation of ASCT to reduce the bias due to patient-related and confounding factors between the groups. Two groups of patients were matched on the logit of the propensity score using calipers of width equal to 0.2 of the standard deviation by the nearest neighbor matching method using the program in EZR version 1.42 (Saitama Medical Center, and Jichi Medical University, Saitama, Japan), which is a graphical user interface for R (The R Foundation for Statistical Computing, Vienna, Austria) (Kanda, 2013).

\section{Statistical analysis}

Fisher's exact test was used to compare differences between categorical variables, whereas the MannWhitney U test was used for continuous or nominal values. PFS and OS were calculated from the date of initial treatment. Kaplan-Meier method was used to create the PFS and OS curves, and differences between the curves were analyzed by the log-rank test. Cox model was used to estimate the hazard ratio with $95 \%$ confidence intervals $(\mathrm{Cl})$ in univariate and multivariate analysis on survival outcomes. Statistical analyses were performed using the program in EZR.

\section{Results}

\section{Patient characteristics}

Among 720 patients who achieved at least stable disease after induction therapy with or without subsequent ASCT, 161 patients of the maintenance group and another 161 patients of the no maintenance group were identified by propensity-score matching and studied. The follow-up periods from the time of diagnosis of these patients ranged from 0.2 to 78.4 months (median, 31.7 months). 
Baseline characteristics of each patient group at diagnosis are summarized in Table 1. The median age was 65 years old (range, 33-89). There were 166 males and 156 females. Patient baseline features including age, gender, performance status (PS), M protein isotype, and the percentages of patients with abnormal laboratory parameters such as hemoglobin, calcium, creatinine, albumin, $\beta 2$-microglobulin, lactate dehydrogenase (LDH), high risk by FISH, and adverse karyotype were not significantly different between the patient groups. The R-ISS stages I, II, and III were distributed in 65 patients $(20.2 \%), 223$ (69.2\%), and 34 (10.6\%), respectively, without significant difference between the groups. 
Table 1

Patient characteristics at diagnosis

\begin{tabular}{|c|c|c|c|c|}
\hline Characteristics & $\begin{array}{l}\text { Maintenance } \\
(n=161)\end{array}$ & $\begin{array}{l}\text { No maintenance } \\
(n=161)\end{array}$ & $\begin{array}{l}\text { Total } \\
(n=322)\end{array}$ & $P$ value \\
\hline Median age (range) & $65(35-89) \mathrm{yr}$ & $65(33-88) y r$ & $65(33-89) \mathrm{yr}$ & 0.88 \\
\hline Gender (M/F) & $84 / 77$ & $82 / 79$ & $166 / 156$ & 0.91 \\
\hline Performance status & $66(46.5 \%)$ & $46(31.1 \%)$ & $112(38.6 \%)$ & 0.06 \\
\hline 0 & $44(31.0 \%)$ & $60(40.4 \%)$ & $104(35.9 \%)$ & \\
\hline 1 & $18(12.7 \%)$ & $18(12.2 \%)$ & $36(12.4 \%)$ & \\
\hline 2 & $9(6.3 \%)$ & $18(12.2 \%)$ & $27(9.3 \%)$ & \\
\hline 3 & $5(3.5 \%)$ & $6(4.1 \%)$ & $11(3.8 \%)$ & \\
\hline \multicolumn{5}{|l|}{4} \\
\hline M protein & $97(60.2 \%)$ & 89 (55.3\%) & $186(57.7 \%)$ & 0.16 \\
\hline $\lg G$ & $22(13.7 \%)$ & $33(20.5 \%)$ & $55(17.1 \%)$ & \\
\hline $\lg \mathrm{A}$ & $1(0.6 \%)$ & $5(3.1 \%)$ & $6(1.9 \%)$ & \\
\hline $\lg D$ & $36(22.4 \%)$ & $32(19.9 \%)$ & $68(21.1 \%)$ & \\
\hline BJP & $5(3.1 \%)$ & $2(1.2 \%)$ & $7(2.2 \%)$ & \\
\hline \multicolumn{5}{|l|}{ Others } \\
\hline Hemoglobin & $86(53.4 \%)$ & $85(52.8 \%)$ & $171(53.1 \%)$ & 1.0 \\
\hline$\geq 10 \mathrm{~g} / \mathrm{dl}$ & $75(46.6 \%)$ & $76(47.2 \%)$ & $151(46.9 \%)$ & \\
\hline \multicolumn{5}{|l|}{$<10 \mathrm{~g} / \mathrm{dl}$} \\
\hline Calcium & $12(7.5 \%)$ & $11(6.8 \%)$ & $23(7.1 \%)$ & 1.0 \\
\hline$>11 \mathrm{mg} / \mathrm{dl}$ & $149(92.5 \%)$ & $150(93.2 \%)$ & 299 (92.9\%) & \\
\hline \multicolumn{5}{|l|}{$\leq 11 \mathrm{mg} / \mathrm{dl}$} \\
\hline Creatinine & $18(11.2 \%)$ & $23(14.3 \%)$ & $41(12.7 \%)$ & 0.51 \\
\hline$>2 \mathrm{mg} / \mathrm{dl}$ & $143(88.8 \%)$ & $138(85.7 \%)$ & $281(87.3 \%)$ & \\
\hline$\leq 2 \mathrm{mg} / \mathrm{dl}$ & & & & \\
\hline
\end{tabular}

LDH: lactate dehydrogenase; FISH: fluorescence in situ hybridization

R-ISS: revised International Staging System; CR: complete response; VGPR: very good partial response; ASCT: autologous stem cell transplantation 


\begin{tabular}{|c|c|c|c|c|}
\hline Characteristics & $\begin{array}{l}\text { Maintenance } \\
(n=161)\end{array}$ & $\begin{array}{l}\text { No maintenance } \\
(n=161)\end{array}$ & $\begin{array}{l}\text { Total } \\
(n=322)\end{array}$ & $P$ value \\
\hline Albumin & $85(52.8 \%)$ & $96(59.6 \%)$ & $181(56.2 \%)$ & 0.26 \\
\hline$\geq 3.5 \mathrm{~g} / \mathrm{dl}$ & $76(47.2 \%)$ & $65(40.4 \%)$ & $141(47.8 \%)$ & \\
\hline \multicolumn{5}{|l|}{$<3.5 \mathrm{~g} / \mathrm{dl}$} \\
\hline ß2-microglobulin & $72(44.7 \%)$ & $65(40.4 \%)$ & $137(42.6 \%)$ & 0.66 \\
\hline$<3.5 \mathrm{mg} / \mathrm{l}$ & $41(25.5 \%)$ & $48(29.8 \%)$ & $89(27.6 \%)$ & \\
\hline $3.5-5.5 \mathrm{mg} / \mathrm{l}$ & $48(29.8 \%)$ & $48(29.8 \%)$ & $96(29.8 \%)$ & \\
\hline \multicolumn{5}{|l|}{$>5.5 \mathrm{mg} / \mathrm{l}$} \\
\hline LDH & $126(78.3 \%)$ & $131(81.4 \%)$ & $257(79.8 \%)$ & 0.58 \\
\hline Normal & $35(21.7 \%)$ & $30(18.6 \%)$ & $65(20.2 \%)$ & \\
\hline \multicolumn{5}{|l|}{ Abnormal } \\
\hline FISH & $104(77.6 \%)$ & $88(80.0 \%)$ & $192(78.7 \%)$ & 0.75 \\
\hline Standard risk & $30(22.4 \%)$ & $22(20.0 \%)$ & $52(21.3 \%)$ & \\
\hline \multicolumn{5}{|l|}{ High risk } \\
\hline Karyotype & $113(73.9 \%)$ & $121(79.6 \%)$ & $234(76.7 \%)$ & 0.28 \\
\hline Normal & $40(26.1 \%)$ & $31(20.4 \%)$ & $71(23.3 \%)$ & \\
\hline \multicolumn{5}{|l|}{ Abnormal } \\
\hline R-ISS stage & $31(19.3 \%)$ & $34(21.1 \%)$ & $65(20.2 \%)$ & 0.91 \\
\hline I & $113(70.2 \%)$ & $110(68.3 \%)$ & $223(69.2 \%)$ & \\
\hline II & $17(10.6 \%)$ & $17(10.6 \%)$ & $34(10.6 \%)$ & \\
\hline \multicolumn{5}{|l|}{ III } \\
\hline Response at front therapy & $65(40.4 \%)$ & $51(31.7 \%)$ & $116(36.0 \%)$ & 0.13 \\
\hline$\geq \mathrm{CR}$ & $96(59.6 \%)$ & $110(68.3 \%)$ & $206(64.0 \%)$ & \\
\hline$\leq$ VGPR & & & & \\
\hline
\end{tabular}

LDH: lactate dehydrogenase; FISH: fluorescence in situ hybridization R-ISS: revised International Staging System; CR: complete response; VGPR: very good partial response; ASCT: autologous stem cell transplantation 


\begin{tabular}{|lllll|}
\hline Characteristics & $\begin{array}{l}\text { Maintenance } \\
(\mathbf{n = 1 6 1 )}\end{array}$ & $\begin{array}{l}\text { No maintenance } \\
(\mathbf{n = 1 6 1 )}\end{array}$ & $\begin{array}{l}\text { Total } \\
(\mathbf{n = 3 2 2})\end{array}$ & P value \\
\hline ASCT & $87(54.0 \%)$ & $87(54.0 \%)$ & $174(54.0 \%)$ & 1.0 \\
Yes & $74(46.0 \%)$ & $74(46.0 \%)$ & $148(46.0 \%)$ & \\
No & & & \\
\hline LDH: lactate dehydrogenase; FISH: fluorescence in situ hybridization & & \\
\hline & & \\
\hline
\end{tabular}

\section{Treatment}

As for induction therapy, most patients were treated with bortezomib-based therapy including bortezomib + cyclophosphamide + dexamethasone (DEX) ( $n=111,34.4 \%)$, bortezomib + DEX ( $n=85,26.4 \%)$, bortezomib + melphalan + prednisolone $(n=53,16.5 \%)$, and bortezomib + doxorubicin + DEX $(n=8,2.5 \%)$, and also with a combination with lenalidomide such as bortezomib + lenalidomide + DEX $(n=29,9.0 \%)$. In contrast, induction with lenalidomide-based therapy was less used and included lenalidomide + DEX ( $\mathrm{n}$ $=16,5.0 \%)$ and a combination with bortezomib $(n=29,9.0 \%)$. Other regimens included melphalan + prednisolone, high-dose DEX, and vincristine + doxorubicin + DEX (Table 2). Between the maintenance and no maintenance groups, the percentages of induction regimen containing bortezomib, lenalidomide, and both were $93.8 \%$ vs $83.8 \%(p=0.0073), 14.9 \%$ vs $13.0 \%(p=0.75)$, and $12.4 \%$ vs $5.6 \%(p=0.05)$, respectively.

Table 2

Induction regimens

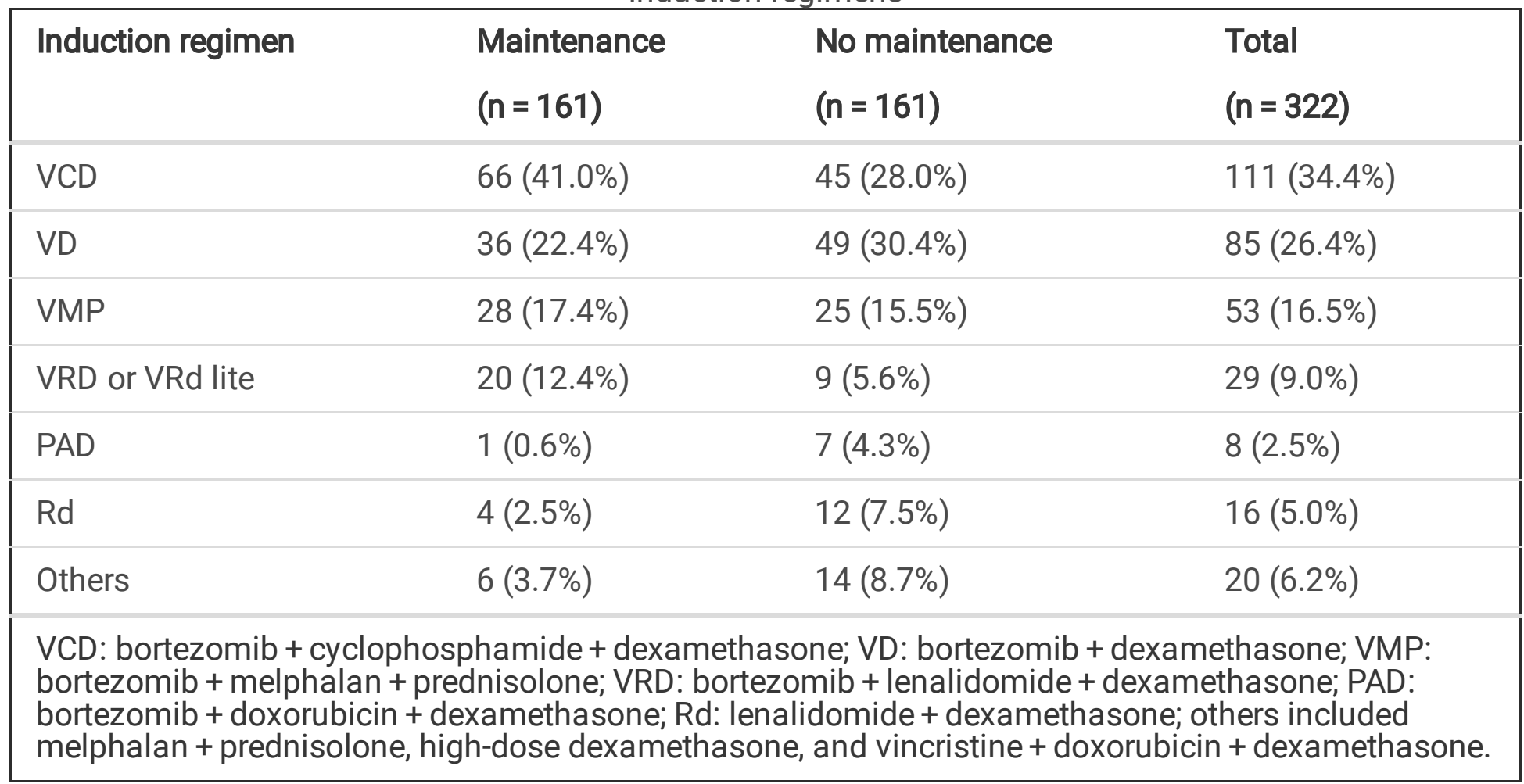


As for the best response obtained after front-line therapy, 116 patients (36.0\%) achieved complete response (CR) or better, and 206 patients $(64.0 \%)$ achieved very good partial response (VGPR) or below. The response rate of $\mathrm{CR}$ or better was higher in the maintenance group ( $40.4 \%$ vs $31.7 \%$ ), but there was no significant difference between the groups $(p=0.13)$. ASCT was performed in 87 patients in each group (54.0\%).

\section{Maintenance/continuous therapy}

Maintenance/continuous regimens were heterogeneous and included IMiDs-based [lenalidomide + DEX $(n=38)$, lenalidomide alone $(n=34)$, elotuzumab + lenalidomide $+\operatorname{DEX}(n=6)$, and thalidomide alone $(n=$ 5)]; PI-based [bortezomib + DEX $(n=18)$, bortezomib alone $(n=17)$, panobinostat + bortezomib + DEX $(n=$ 1), ixazomib alone $(n=9)$, carfilzomib $+\operatorname{DEX}(n=2)$, and bortezomib + melphalan + prednisolone $(n=1)]$; combination of IMiDs and PI-based [bortezomib + lenalidomide + DEX $(n=10)$, bortezomib + thalidomide $+\operatorname{DEX}(n=6)$, carfilzomib + lenalidomide + DEX $(n=6)$, ixazomib + lenalidomide + DEX $(n=6)$, and bortezomib + thalidomide $(n=1)]$; and DEX alone $(n=1)$.

\section{Progression-free survival according to maintenance therapy}

We first compared PFS between the maintenance group and the matched no maintenance group. PFS was significantly prolonged in the maintenance group compared with the no maintenance group (median, 37.7 and 21.9 months, respectively, $p=0.0002$, Fig. $1 \mathrm{~A}$ ). In terms of risk stratification by cytogenetic abnormalities by FISH, the median PFS was 39.7 and 21.1 months in standard-risk patients $(p<0.00001$, Fig. 1B), and was 25.7 and 18.8 months in high-risk patients ( $p=0.42$, Fig. $1 \mathrm{C})$, respectively. According to the R-ISS stage, significant difference in PFS was observed in patients with stage I ( $p=0.037$, Fig. 1D) and II ( $p=0.00094$, Fig. 1E) by maintenance therapy, but not in patients with stage III disease $(p=0.37$, Fig. 1F). As for the response status before maintenance therapy, PFS was longer in patients who achieved CR or better than that in those who did not achieve CR, but significant difference in PFS by maintenance was observed only in $\leq$ VGPR patients $(p=0.0018$, Fig. $1 \mathrm{H})$ but not in $\geq$ CR patients $(p=$ 0.27, Fig. 1G). As for ASCT, prolongation of PFS was observed in both non-transplanted ( $p=0.0008$, Fig. 1I) and transplanted patients $(p=0.017$, Fig. $1 \mathrm{~J})$.

According to different maintenance of the PI-based, IMiDs-based, and combination of PI + IMiDs-based regimens, the median PFS were 23.4, 37.7, and 48.3 months, respectively, and was longer in the combination with $\mathrm{PI}+\mathrm{IMiDs}$-based than $\mathrm{PI}$ or IMiDs alone groups in the whole maintenance cohort $\mathrm{p}=$ 0.021 , Fig. $1 \mathrm{~K}$ ). In terms of cytogenetic risk groups by FISH, the median PFS were 22.9 months, 39.0 months, and not reached in standard-risk patients $(p=0.16$, Supplementary Fig. $1 \mathrm{~A})$, and 15.0, 18.4, and 48.3 months in high-risk patients ( $p=0.088$, Supplementary Fig. 1B) according to maintenance therapy with PI-based, IMiDs-based, or the combination of PI + IMiDs-based, respectively. In comparison of the PIor IMiDs-based vs PI + IMiDs-based regimens, the median PFS was 39.0 months and not reached in standard-risk patients $(p=0.094)$, and 18.4 and 48.3 months in high-risk patients $(p=0.030)$, respectively. Thus, maintenance with combination of PI and IMiD is likely to prolong PFS regardless of risk category by FISH. As for the effect of ASCT, the median PFS of non-transplanted patients were 18.0 months, 35.4 
months, and not reached ( $p=0.077$, Supplementary Fig. 1C) and those of transplanted patients were not reached, 38.7 months, and 48.3 months ( $p=0.69$, Supplementary Fig. 1D) according to maintenance therapy with PI, IMiDs, or the combination of PI + IMiDs, respectively. In comparison of the PI- or IMiDsbased vs PI + IMiDs-based regimens, the median PFS was 24.1 months and not reached in nontransplanted patients $(p=0.063)$, and 39.7 and 48.3 months in transplanted patients $(p=0.55)$, respectively. Thus, maintenance therapy with combination of PI + IMiDs is likely to extend the PFS in nontransplanted patients although there was no statistically significant difference.

\section{Overall survival according to maintenance therapy}

The median OS was not reached in either group and there was no significant difference in OS by maintenance/continuous therapy ( $p=0.19$, Fig. $2 \mathrm{~A}$ ). In terms of cytogenetic risk by FISH, OS was not different between the maintenance vs no maintenance groups irrespective of risk status [standard risk ( $p$ $=0.36$, Fig. $2 B$ ) and high risk ( $p=0.21$, Fig. $2 C$ ) ]. Lack of OS difference between maintenance vs no maintenance was also noted in each of the R-ISS stages [stage I $(p=0.14$, Fig. 2D); stage II $(p=0.17$, Fig. 2E); and stage III ( $p=0.30$, Fig. $2 \mathrm{~F})]$. As for the response status before maintenance/continuous therapy, OS appeared to be better in the maintenance group when response was $\leq$ VGPR, but maintenance therapy did not provide statistically significant benefit irrespective of response status before maintenance/continuous therapy $[\geq$ CR patients $(p=0.28$, Fig. $2 G)$ and $\leq$ VGPR patients $(p=0.053$, Fig. $2 \mathrm{H}$ )]. As for ASCT, OS appeared to be better in non-transplanted patients by continuous therapy, but there was no significant difference in non-transplanted patients $(p=0.13$, Fig. $2 \mathrm{l})$ and transplanted patients ( $p=0.57$, Fig. $2 \mathrm{~J})$.

According to the different maintenance regimens, OS was better in both PI-based and combination of PI + IMiDs-based groups, but the difference in OS between 3 maintenance groups was not statistically significant ( $p=0.35$, Fig. $2 \mathrm{~K}$ ). We compared the differences in OS in terms of cytogenetic risk or implementation of ASCT, but there was no significant difference between 3 maintenance groups (Supplementary Fig. 2).

\section{Univariate and multivariate analysis}

Next, the statistical significance of risk factors related to PFS and OS was evaluated by univariate and multivariate analysis. As shown in Table 3 , age ( $\geq 65$ years old), R-ISS stage III, and non-CR response after initial therapy were significant poor prognostic factors for PFS, whereas implementation of ASCT as well as maintenance/continuous therapy were significant favorable factors for PFS. In multivariate analysis, non-CR response after initial therapy, and implementation of ASCT, as well as maintenance therapy were significant independent prognostic factors for PFS. 
Table 3

Univariate and multivariate analysis for progression-free survival

\begin{tabular}{|c|c|c|c|c|c|c|}
\hline \multirow[t]{2}{*}{ Variable } & \multicolumn{3}{|c|}{ Univariate } & \multicolumn{3}{|c|}{ Multivariate* } \\
\hline & HR & $95 \% \mathrm{Cl}$ & $P$ value & HR & $95 \% \mathrm{Cl}$ & $P$ value \\
\hline Age [ $\geq 65$ years] & 1.542 & $1.128-2.109$ & 0.0067 & 0.850 & $0.563-1.284$ & 0.44 \\
\hline Gender [Male] & 1.028 & $0.756-1.399$ & 0.86 & - & - & - \\
\hline R-ISS [stage III] & 1.718 & $1.085-2.719$ & 0.021 & 1.468 & $0.923-2.336$ & 0.11 \\
\hline Response [non-CR] & 2.376 & $1.650-3.421$ & $<0.0001$ & 1.914 & $1.313-2.788$ & 0.00073 \\
\hline ASCT & 0.445 & $0.325-0.607$ & $<0.0001$ & 0.428 & $0.282-0.648$ & $<0.0001$ \\
\hline Maintenance & 0.562 & $0.413-0.766$ & 0.00026 & 0.529 & $0.386-0.725$ & $<0.0001$ \\
\hline \multicolumn{7}{|c|}{ *Variables significance at $p<0.1$ in the univariate model were entered in the multivariate model. } \\
\hline
\end{tabular}

As for OS, age ( $\geq 65$ years old), R-ISS stage III, and non-CR response after initial therapy were significant unfavorable prognostic factors for OS. Implementation of ASCT was a significant favorable factor for OS but administration of maintenance/continuous therapy was not (Table 4). In multivariate analysis, R-ISS stage III, non-CR response after initial therapy, and implementation of ASCT were significant independent prognosis factors for OS (Table 4).

Table 4

Univariate and multivariate analysis for overall survival

\begin{tabular}{|c|c|c|c|c|c|c|}
\hline \multirow[t]{2}{*}{ Variable } & \multicolumn{3}{|c|}{ Univariate } & \multicolumn{3}{|c|}{ Multivariate } \\
\hline & HR & $95 \% \mathrm{Cl}$ & $P$ value & HR & $95 \% \mathrm{Cl}$ & $P$ value \\
\hline Age $[\geq 65$ years $]$ & 2.237 & $1.362-3.675$ & 0.0015 & 1.223 & $0.647-2.313$ & 0.53 \\
\hline Gender [Male] & 0.847 & $0.531-1.351$ & 0.49 & - & - & - \\
\hline R-ISS [stage III] & 2.774 & $1.541-4.994$ & 0.00067 & 2.699 & $1.495-4.871$ & 0.00098 \\
\hline Response [non-CR] & 2.775 & $1.543-4.990$ & 0.00065 & 2.120 & $1.160-3.876$ & 0.015 \\
\hline ASCT & 0.348 & $0.212-0.571$ & $<0.0001$ & 0.475 & $0.250-0.903$ & 0.023 \\
\hline Maintenance & 0.731 & $0.458-1.165$ & 0.19 & - & - & - \\
\hline \multicolumn{7}{|c|}{ *Variables significance at $p<0.1$ in the univariate model were entered in the multivariate model. } \\
\hline $\begin{array}{l}\text { R-ISS: revised Intern } \\
\text { autologous stem ce }\end{array}$ & $\begin{array}{l}\text { lal St } \\
\text { ispla }\end{array}$ & $\begin{array}{l}\text { g System; Res } \\
\text { ion; Maintenar }\end{array}$ & $\begin{array}{l}\text { se: resp } \\
\text { maintel }\end{array}$ & $\begin{array}{l}\text { at } \mathrm{fr} \\
\text { eor } \mathrm{c}\end{array}$ & $\begin{array}{l}\text { ine therapy; AS } \\
\text { nuous therapy }\end{array}$ & \\
\hline
\end{tabular}


We retrospectively evaluated the efficacy of maintenance/continuous therapy by propensity-score matched analysis using the cohort data of the JSM studies. We have demonstrated that maintenance therapy significantly improved PFS but not OS in both transplant-eligible and -ineligible MM patients. Our results of PFS benefit by maintenance were consistent with those of previously reported as clinical trials (Attal et al, 2012; Dimopoulos et al, 2019; Dimopoulos et al, 2020b; Goldschmidt et al, 2018; Jackson et al, 2019; McCarthy et al, 2012; Morgan et al, 2012; Palumbo et al, 2014a; Palumbo et al, 2010; Palumbo et al, 2014b; Palumbo et al, 2012; Sonneveld et al, 2012) and routine practice in transplant-eligible patients (Chakraborty et al, 2018; Huang et al, 2018; Jagannath et al, 2018; Sivaraj et al, 2017), and further provide evidence of similar PFS benefit for transplant-ineligible patients as well in real world settings. Regarding the OS, most of the clinical trials have failed to show the benefit of maintenance therapy on OS except for some limited studies (McCarthy et al, 2012; Morgan et al, 2012; Palumbo et al, 2014b), and thus, OS benefits of maintenance therapy have still been controversial (Dimopoulos et al, 2020a). In general, OS benefit is influenced by the efficacy of induction and salvage therapy, and accordingly, different results may have been due to differences in treatment regimens and durations, and in prognostic variables not properly assessed. Highly effective drugs with novel mechanisms such as daratumumab have emerged and are incorporated into clinical practice as salvage therapy (Dimopoulos et al, 2016; Palumbo et al, 2016), and more recently, as front-line therapy as well as continuous therapy, showing the excellent results in PFS and OS (Facon et al, 2019; Mateos et al, 2020; Mateos et al, 2018). Thus, the present study should be interrupted as evidence before the era of daratumumab in both transplant-eligible and ineligible patients in clinical practice.

In a subgroup analysis, the benefit of maintenance/continuous therapy was more significant in patients who did not achieve CR than those who had achieved CR or better after induction therapy. In addition, with regard to the cytogenetic abnormalities and R-ISS stage, the benefit of maintenance therapy in PFS was more notable in patients with cytogenetic standard risk or R-ISS stage I and II than those with cytogenetic high risk or R-ISS stage III. Thus, our results would indicate that there might be no additional effects of subsequent maintenance/continuous therapy in patients who had already achieved a deep response, and such therapy is most beneficial to patients with standard risk or R-ISS stage I and II. In fact, the benefit of maintenance therapy by lenalidomide or ixazomib was more apparent in PR patients than in CR patients of Myeloma XI and TOURMALINE-MM3 trials (Dimopoulos et al, 2019; Jackson et al, 2019). Moreover, OS benefit by lenalidomide maintenance post ASCT was less significant in patients with the ISS stage III by meta-analysis (McCarthy et al, 2017). Therefore, maintenance/continuous therapy appeared to exert benefit to patients with cytogenetic standard risk and R-ISS stages I and II, especially when deep response was not achieved by induction therapy.

With regard to the relationship between the depth of response and the treatment outcome, recent randomized clinical trials have shown almost the consistently superior PFS and OS in patients who achieved minimal residual disease (MRD) negativity, regardless of treatment regimens (4 drugs or 3 drugs) (Mateos et al, 2020) and even with or without ASCT (Perrot et al, 2018). A large meta-analysis has confirmed the significance of MRD negativity as the most relevant surrogate marker not only for PFS but also for OS (Munshi et al, 2020). Taken together, these results suggest that the long-term outcome 
depends on the susceptibility of MM cells to any treatment. In this study, median PFS and OS were longer in $\geq$ CR patients than $\leq$ VGPR patients, but additional benefit of maintenance therapy was not observed in $\geq \mathrm{CR}$ patients. Therefore, it would be necessary to develop more appropriate treatment strategy in patients with $\mathrm{CR}$ and MRD negativity to further control the disease status and eradicate the minimal residual disease if any.

In multivariate analysis, response of CR or better and implementation of upfront ASCT were independent favorable factors for both PFS (Table 3) and OS (Table 4), whereas maintenance therapy was an independent favorable factor for PFS alone and so was R-ISS stage for OS alone. These results indicate that maintenance therapy was useful in the short-term outcome such as prolongation of PFS but not for the long-term outcome such as OS that depends on the most crucial factors including deep response as $\geq \mathrm{CR}$, implementation of ASCT, and R-ISS stage, leaving maintenance therapy less useful. More importantly, our results did not even observe PFS benefits by maintenance therapy in patients with cytogenetic high risk or R-ISS stage III. Novel treatment strategies besides maintenance/continuous therapy are needed to further improve the outcome specifically in high-risk patients in clinical practice.

The righteousness of the concept of long-term/continuous treatment is now widely recognized; however, clinical issues regarding treatment factors such as optimal regimen and treatment duration, and tumor factors including treatment sensitivity remain to be elucidated. With regard to therapeutic regimens, IMiDs and Pls have been studied extensively from the perspective of convenience and feasibility. Among them, lenalidomide-based maintenance has been confirmed as the most effective regimen by a network metaanalysis (Gay et al, 2018). In contrast, bortezomib rather than lenalidomide maintenance appeared to be more effective in high-risk patients (Chakraborty et al, 2018; Sivaraj et al, 2017). Several studies have shown the effectiveness of combination with IMiDs and Pls as maintenance/continuous therapy, for example, thalidomide + bortezomib, lenalidomide + bortezomib, or lenalidomide + carfilzomib (Durie et al, 2017; Durie et al, 2020; Gay et al, 2020; Palumbo et al, 2014a; Palumbo et al, 2010). Recently, a large cohort study of highly effective regimen such as bortezomib + lenalidomide + DEX induction and riskadapted maintenance approach has shown the significant benefits of maintenance with $\mathrm{PI}+$ IMiDs in PFS and OS, especially in high-risk patients (Joseph et al, 2020). Our results have also suggested that the combination with Pls and IMiDs were more beneficial than PI or IMiDs alone. Furthermore, ongoing trials have included daratumumab not only for induction therapy but also for maintenance, showing particularly encouraging results (Moreau et al, 2019; Voorhees et al, 2020). The efficacy and feasibility of these intensive approaches should be clarified in clinical practice.

The present study has several limitations because of its retrospective nature and the number of patients became limited due to the application of the propensity-score matched methods. The therapeutic regimens, timing, and duration of maintenance/continuous therapy varied because of the discretion of the attending physicians according to the treatment policy of each facility. Therefore, survival outcomes were evaluated from the start of initial treatment, and the duration and efficacy of maintenance itself could not be analyzed. Because of the wide variety of drug combinations, we categorized the maintenance/continuous therapy into 3 groups such as PI-based, IMiDs-based, and PI + IMiDs-based 
regimens, but it was not possible to compare the efficacy of each maintenance regimen. Also, induction therapy as front-line treatment was quite heterogeneous to be classified as bortezomib- and/or lenalidomide-based regimens, and the percentage of induction regimen containing bortezomib and lenalidomide was higher in the maintenance group than in no maintenance group. In this regard, the response rate of $\mathrm{CR}$ or better after front-line therapy was higher in the maintenance group than in no maintenance group, but there was no significant difference in the response rate of CR or better. In addition, no MRD data were available in this analysis and it is unclear whether maintenance therapy was effective in MRD-negative patients in clinical practice. Finally, we had not collected safety data of maintenance/continuous therapy. The balance between efficacy and feasibility should be evaluated to prevent over treatment during the maintenance/continuous therapy in the future.

In conclusion, our results have demonstrated that maintenance or continuous treatment were associated with a reduced risk of progression in both transplant-eligible and -ineligible patients, especially in patients with standard risk and/or suboptimal response to induction therapy. Although there were no statistically significant differences in OS by maintenance therapy, these approaches tended to improve OS in nontransplant patients and in those with suboptimal response. Regarding the maintenance regimens, combination therapy of $\mathrm{PI}+$ IMiDs appeared to be more appropriate than PI or IMiDs alone. Therefore, the results of our study support the continuation of current maintenance approach in routine clinical practice in the management of MM.

\section{Declarations}

\section{Acknowledgments}

The collaborators in this study are listed in the Supplementary Appendix.

\section{Author contribution}

All authors contributed to the study conception and design. SO, HM, and KS designed the research study. Data collection and analysis were performed by SO, HH, HK, TS, KS, TI, KS, TN, SI, YN, KS, and NN. The first draft of the manuscript was written by $\mathrm{SO}$ and $\mathrm{KS}$, and all authors commented on previous versions of the manuscript. All authors read and approved the final manuscript.

\section{Conflict of interest}

Dr. Kazutaka Sunami has received research funding from Ono Pharmaceutical Co. Ltd., Takeda Pharmaceutical Co. Ltd., Novartis Pharmaceutical Co. Ltd., and Celgene Co. Ltd. All other authors declare no conflict of interest.

\section{References}


1. Attal M, Lauwers-Cances V, Marit G, Caillot D, Moreau P, Facon T, et al (2012) Lenalidomide maintenance after stem-cell transplantation for multiple myeloma. N Engl J Med 366:1782-1791.

2. Benboubker L, Dimopoulos MA, Dispenzieri A, Catalano J, Belch AR, Cavo M, et al (2014) Lenalidomide and dexamethasone in transplant-ineligible patients with myeloma. N Engl J Med 371: 906-917.

3. Chakraborty R, Muchtar E, Kumar SK, Buadi FK, Dingli D, Dispenzieri A, et al (2018) Outcomes of maintenance therapy with lenalidomide or bortezomib in multiple myeloma in the setting of early autologous stem cell transplantation. Leukemia 32: 712-718.

4. Dimopoulos MA, Gay F, Schjesvold F, Beksac M, Hajek R, Weisel KC, et al (2019) Oral ixazomib maintenance following autologous stem cell transplantation (TOURMALINE-MM3): a double-blind, randomised, placebo-controlled phase 3 trial. Lancet 393: 253-264.

5. Dimopoulos MA, Jakubowiak AJ, McCarthy PL, Orlowski RZ, Attal M, Bladé J, et al (2020a) Developments in continuous therapy and maintenance treatment approaches for patients with newly diagnosed multiple myeloma. Blood Cancer J 10:17.

6. Dimopoulos MA, Oriol A, Nahi H, San-Miguel J, Bahlis NJ, Usmani SZ, et al (2016) Daratumumab, Lenalidomide, and Dexamethasone for Multiple Myeloma. N Engl J Med 375: 1319-1331.

7. Dimopoulos MA, Špička I, Quach H, Oriol A, Hájek R, Garg M, et al (2020b) Ixazomib as postinduction maintenance for patients with newly diagnosed multiple myeloma not undergoing autologous stem cell transplantation: The phase III TOURMALINE-MM4 trial. J Clin Oncol 38: 4030-4041.

8. Durie BG, Harousseau JL, Miguel JS, Blade J, Barlogie B, Anderson K, et al (2006) International uniform response criteria for multiple myeloma. Leukemia 20: 1467-1473.

9. Durie BG, Hoering A, Abidi MH, Rajkumar SV, Epstein J, Kahanic SP, et al (2017) Bortezomib with lenalidomide and dexamethasone versus lenalidomide and dexamethasone alone in patients with newly diagnosed myeloma without intent for immediate autologous stem-cell transplant (SWOG S0777): a randomised, open-label, phase 3 trial. Lancet 389: 519-527.

10. Durie BGM, Hoering A, Sexton R, Abidi MH, Epstein J, Rajkumar SV, et al (2020) Longer term follow-up of the randomized phase III trial SWOG S0777: bortezomib, lenalidomide and dexamethasone vs. lenalidomide and dexamethasone in patients (Pts) with previously untreated multiple myeloma without an intent for immediate autologous stem cell transplant (ASCT). Blood Cancer J 10: 53.

11. Facon T, Dimopoulos MA, Dispenzieri A, Catalano JV, Belch A, Cavo M, et al (2018) Final analysis of survival outcomes in the phase 3 FIRST trial of up-front treatment for multiple myeloma. Blood 131: 301-310.

12. Facon T, Kumar S, Plesner T, Orlowski RZ, Moreau P, Bahlis N, et al (2019) Daratumumab plus lenalidomide and dexamethasone for untreated myeloma. N Engl J Med 380: 2104-2115.

13. Gay F, Jackson G, Rosinol L, Holstein SA, Moreau P, Spada S, et al (2018) Maintenance treatment and survival in patients with myeloma: a systematic review and network meta-analysis. JAMA Oncology 4: 1389-1397. 
14. Gay F, Musto P, Rota Scalabrini D, Galli M, Belotti A, Zamagni E, et al (2020) Survival analysis of newly diagnosed transplant-eligible multiple myeloma patients in the randomized Forte trial. Blood 136 (suppl 1): 35-37.

15. Goldschmidt H, Lokhorst HM, Mai EK, van der Holt B, Blau IW, Zweegman S, et al (2018) Bortezomib before and after high-dose therapy in myeloma: long-term results from the phase III HOVON65/GMMG-HD4 trial. Leukemia 32: 383-390.

16. Huang J, Phillips S, Byrne M, Chinratanalab W, Engelhardt BG, Goodman SA, et al (2018) Lenalidomide vs bortezomib maintenance choice post-autologous hematopoietic cell transplantation for multiple myeloma. Bone Marrow Transplant 53: 701-707.

17. International Myeloma Working Group (2003) Criteria for the classification of monoclonal gammopathies, multiple myeloma and related disorders: a report of the International Myeloma Working Group. Br J Haematol 121: 749-757.

18. Jackson GH, Davies FE, Pawlyn C, Cairns DA, Striha A, Collett C, et al (2019) Lenalidomide maintenance versus observation for patients with newly diagnosed multiple myeloma (Myeloma XI): a multicentre, open-label, randomised, phase 3 trial. Lancet Oncol 20: 57-73.

19. Jagannath S, Abonour R, Durie BGM, Narang M, Terebelo HR, Gasparetto CJ, et al (2018) Impact of post-ASCT maintenance therapy on outcomes in patients with newly diagnosed multiple myeloma in Connect MM. Blood Adv 2: 1608-1615.

20. Joseph NS, Kaufman JL, Dhodapkar MV, Hofmeister CC, Almaula DK, Heffner LT, et al (2020) Longterm follow-up results of lenalidomide, bortezomib, and dexamethasone induction therapy and riskadapted maintenance approach in newly diagnosed multiple myeloma. J Clin Oncol 38: 1928-1937.

21. Kanda Y (2013) Investigation of the freely available easy-to-use software 'EZR' for medical statistics. Bone Marrow Transplant 48: 452-458.

22. Kumar SK, Dispenzieri A, Lacy MQ, Gertz MA, Buadi FK, Pandey S, et al (2014) Continued improvement in survival in multiple myeloma: changes in early mortality and outcomes in older patients. Leukemia 28: 1122-1128.

23. Kumar SK, Rajkumar SV, Dispenzieri A, Lacy MQ, Hayman SR, Buadi FK, et al (2008) Improved survival in multiple myeloma and the impact of novel therapies. Blood 111:2516-2520.

24. Mateos MV, Cavo M, Blade J, Dimopoulos MA, Suzuki K, Jakubowiak A, et al (2020) Overall survival with daratumumab, bortezomib, melphalan, and prednisone in newly diagnosed multiple myeloma (ALCYONE): a randomised, open-label, phase 3 trial. Lancet 395: 132-141.

25. Mateos MV, Dimopoulos MA, Cavo M, Suzuki K, Jakubowiak A, Knop S, et al (2018) Daratumumab plus bortezomib, melphalan, and prednisone for untreated myeloma. N Engl J Med 378: 518-528.

26. McCarthy PL, Holstein SA, Petrucci MT, Richardson PG, Hulin C, Tosi P, et al (2017) Lenalidomide maintenance after autologous stem-cell transplantation in newly diagnosed multiple myeloma: a meta-analysis. J Clin Oncol 35: 3279-3289.

27. McCarthy PL, Owzar K, Hofmeister CC, Hurd DD, Hassoun H, Richardson PG, et al (2012) Lenalidomide after stem-cell transplantation for multiple myeloma. N Engl J Med 366: 1770-1781. 
28. Moreau P, Attal M, Hulin C, Arnulf B, Belhadj K, Benboubker L, et al (2019) Bortezomib, thalidomide, and dexamethasone with or without daratumumab before and after autologous stem-cell transplantation for newly diagnosed multiple myeloma (CASSIOPEIA): a randomised, open-label, phase 3 study. Lancet 394: 29-38.

29. Morgan GJ, Gregory WM, Davies FE, Bell SE, Szubert AJ, Brown JM, et al (2012) The role of maintenance thalidomide therapy in multiple myeloma: MRC Myeloma IX results and meta-analysis. Blood 119: 7-15.

30. Munshi NC, Avet-Loiseau H, Anderson KC, Neri P, Paiva B, Samur M, et al (2020) A large metaanalysis establishes the role of MRD negativity in long-term survival outcomes in patients with multiple myeloma. Blood Adv 4: 5988-5999.

31. Ozaki S, Handa H, Saitoh T, Murakami H, Itagaki M, Asaoku H, et al (2015) Trends of survival in patients with multiple myeloma in Japan: a multicenter retrospective collaborative study of the Japanese Society of Myeloma. Blood Cancer J 5: e349.

32. Palumbo A, Avet-Loiseau H, Oliva S, Lokhorst HM, Goldschmidt H, Rosinol L, et al (2015a) Revised International Staging System for multiple myeloma: A report from International Myeloma Working Group. J Clin Oncol 33: 2863-2869.

33. Palumbo A, Bringhen S, Larocca A, Rossi D, Di Raimondo F, Magarotto V, et al (2014a) Bortezomibmelphalan-prednisone-thalidomide followed by maintenance with bortezomib-thalidomide compared with bortezomib-melphalan-prednisone for initial treatment of multiple myeloma: updated follow-up and improved survival. J Clin Oncol 32: 634-640.

34. Palumbo A, Bringhen S, Rossi D, Cavalli M, Larocca A, Ria R, et al (2010) Bortezomib-melphalanprednisone-thalidomide followed by maintenance with bortezomib-thalidomide compared with bortezomib-melphalan-prednisone for initial treatment of multiple myeloma: a randomized controlled trial. J Clin Oncol 28: 5101-5109.

35. Palumbo A, Cavallo F, Gay F, Di Raimondo F, Ben Yehuda D, Petrucci MT, et al (2014b) Autologous transplantation and maintenance therapy in multiple myeloma. N Engl J Med 371: 895-905.

36. Palumbo A, Chanan-Khan A, Weisel K, Nooka AK, Masszi T, Beksac M, et al (2016) Daratumumab, bortezomib, and dexamethasone for multiple myeloma. N Engl J Med 375: 754-766.

37. Palumbo A, Gay F, Cavallo F, Di Raimondo F, Larocca A, Hardan I, et al (2015b) Continuous therapy versus fixed duration of therapy in patients with newly diagnosed multiple myeloma. J Clin Oncol 33: 3459-3466.

38. Palumbo A, Hajek R, Delforge M, Kropff M, Petrucci MT, Catalano J, et al (2012) Continuous lenalidomide treatment for newly diagnosed multiple myeloma. N Engl J Med 366: 1759-1769.

39. Perrot A, Lauwers-Cances V, Corre J, Robillard N, Hulin C, Chretien ML, et al (2018) Minimal residual disease negativity using deep sequencing is a major prognostic factor in multiple myeloma. Blood 132: 2456-2464.

40. Rajkumar SV (2020) Multiple myeloma: 2020 update on diagnosis, risk-stratification and management. Am J Hematol 95: 548-567. 
41. Rajkumar SV, Dimopoulos MA, Palumbo A, Blade J, Merlini G, Mateos MV, et al (2014) International Myeloma Working Group updated criteria for the diagnosis of multiple myeloma. Lancet Oncol 15: e538-e548.

42. Sivaraj D, Green MM, Li Z, Sung AD, Sarantopoulos S, Kang Y, et al (2017) Outcomes of maintenance therapy with bortezomib after autologous stem cell transplantation for patients with multiple myeloma. Biol Blood Marrow Transplant 23: 262-268.

43. Sonneveld P, Schmidt-Wolf IG, van der Holt B, El Jarari L, Bertsch U, Salwender H, et al (2012) Bortezomib Induction and Maintenance Treatment in Patients With Newly Diagnosed Multiple Myeloma: Results of the Randomized Phase III HOVON-65/ GMMG-HD4 Trial. J Clin Oncol 30: 29462955.

44. Voorhees PM, Kaufman JL, Laubach J, Sborov DW, Reeves B, Rodriguez C, et al (2020) Daratumumab, lenalidomide, bortezomib, and dexamethasone for transplant-eligible newly diagnosed multiple myeloma: the GRIFFIN trial. Blood 136: 936-945.

\section{Figures}


Figure 1
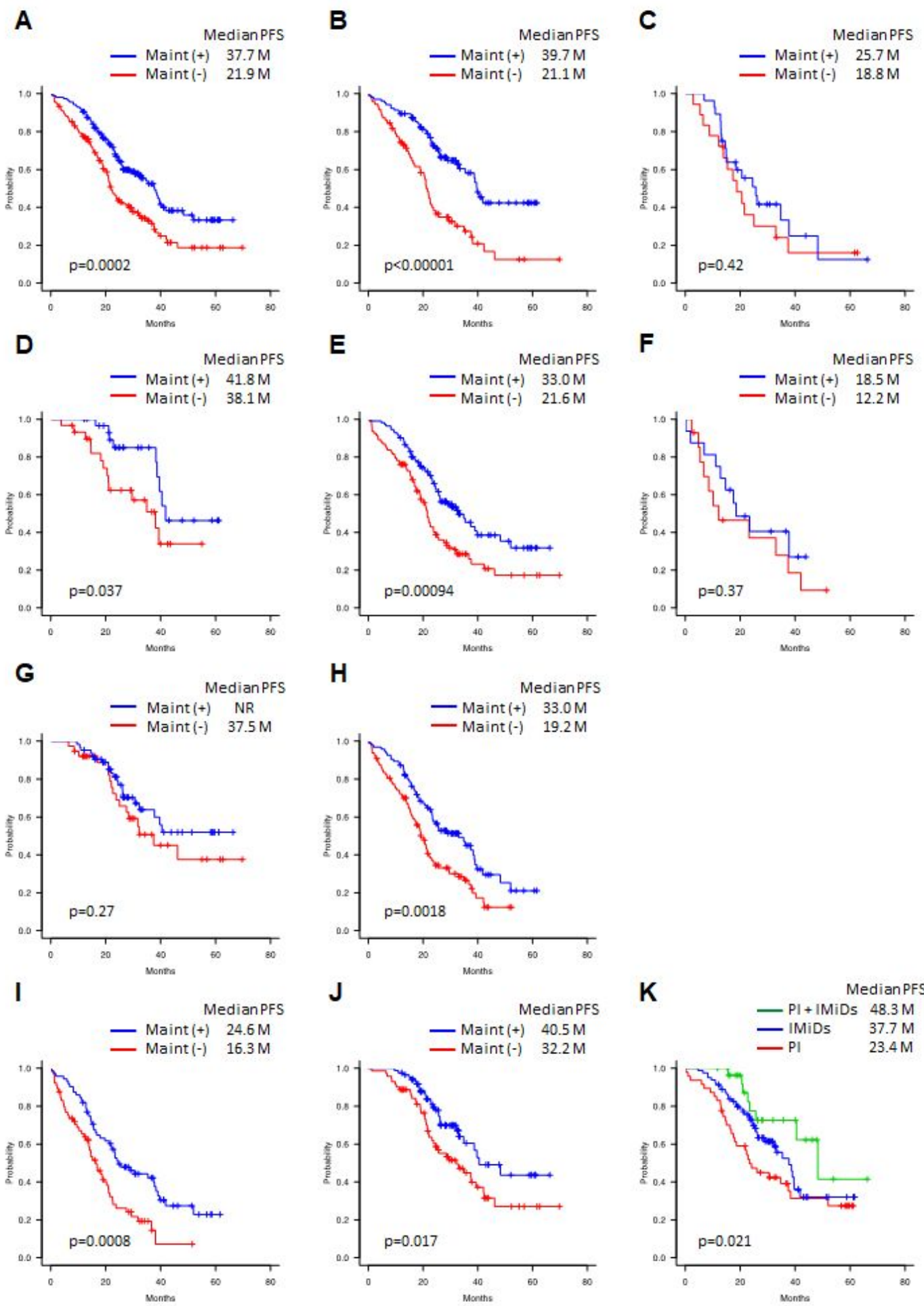

Figure 1

Efficacy of maintenance therapy on PFS in each setting PFS in total patients (A); patients with standard risk (B) or high risk (C) by FISH; patients with R-ISS stage I (D), stage II (E), and stage III (F); patients who achieved $\geq$ complete response $(G)$ or $\leq$ very good partial response $(H)$ after induction therapy; patients treated without upfront ASCT (I) or with ASCT (J). PFS according to the maintenance regimen (K). Maint, 
maintenance/continuous therapy; PI, proteasome inhibitors; IMiDs, immunomodulatory drugs, M, months; NR, not reached.

Figure 2
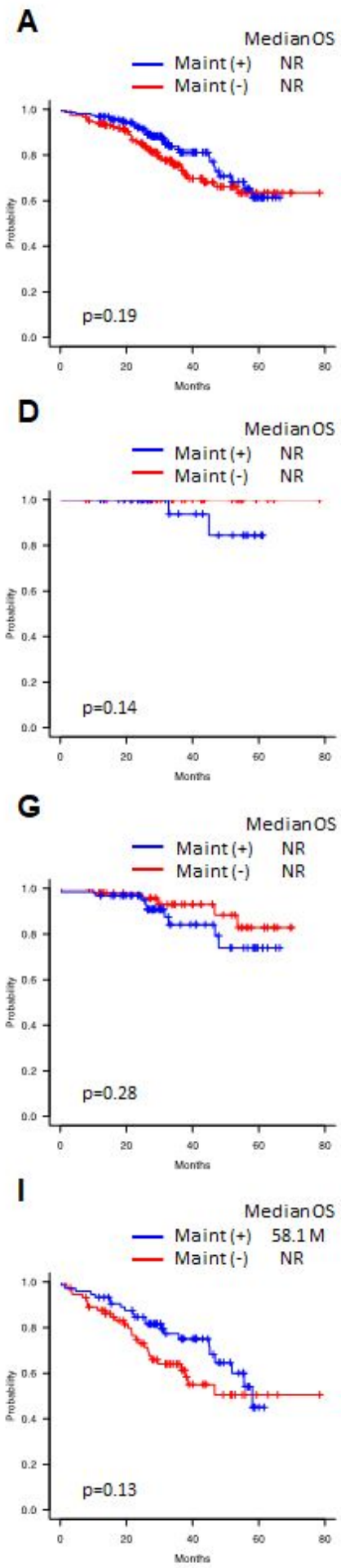

B

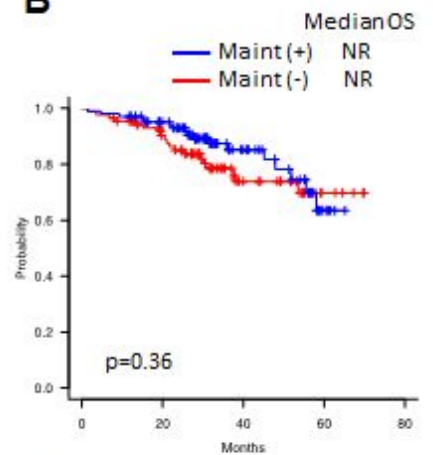

$\mathbf{E}$
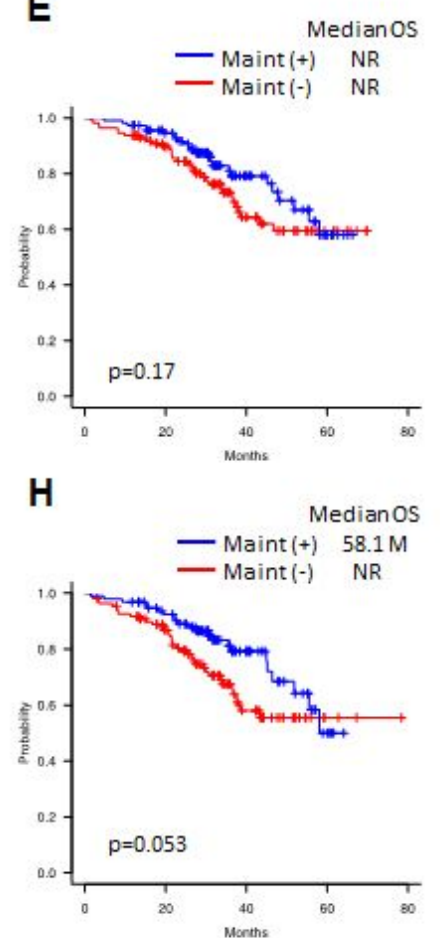

$\mathbf{J}$

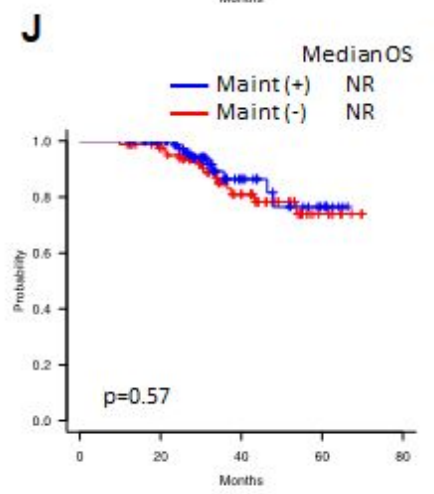

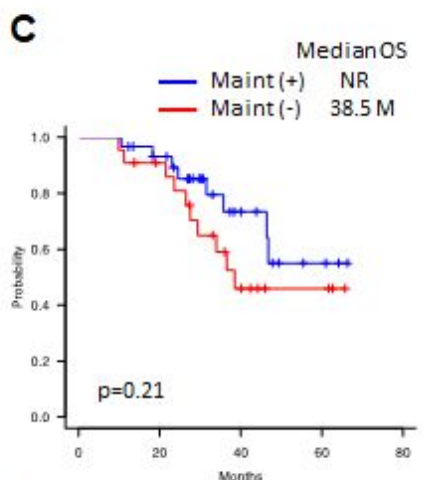

$\mathbf{F}$

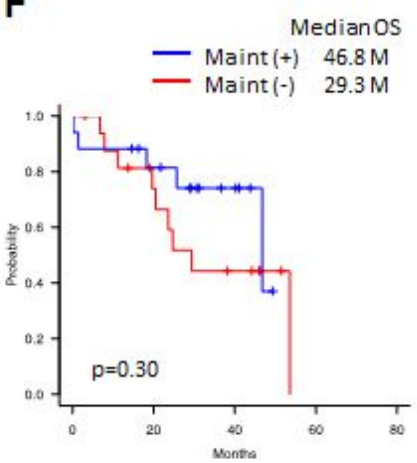

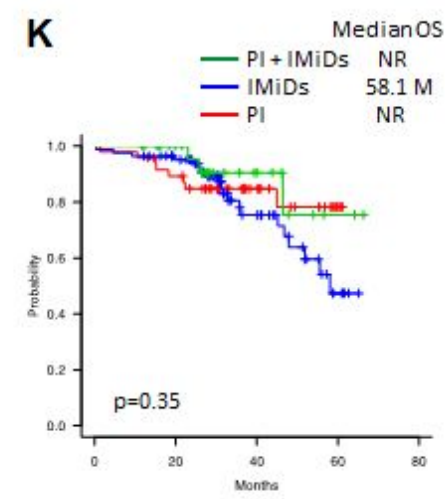

Figure 2

Efficacy of maintenance therapy on OS in each setting OS in total patients (A); patients with standard risk (B) or high risk (C) by FISH; patients with R-ISS stage I (D), stage II (E), and stage III (F); patients who achieved $\geq$ complete response $(G)$ or $\leq$ very good partial response $(H)$ after induction therapy; patients 
treated without upfront ASCT (I) or with ASCT $(\mathrm{J})$. OS according to the maintenance regimen $(\mathrm{K})$. Maint, maintenance/continuous therapy; $\mathrm{Pl}$, proteasome inhibitors; IMiDs, immunomodulatory drugs, $\mathrm{M}$, months; NR, not reached.

\section{Supplementary Files}

This is a list of supplementary files associated with this preprint. Click to download.

- Suppl.pptx 